\title{
Quantization of the QCD string with a helical structure
}

\author{
Šárka Todorova-Nová \\ Institute of Particle and Nuclear Physics, Charles University, Pragu®
}

\begin{abstract}
The quantum properties of a helix-like shaped QCD string are studied in the context of the semi-classical Lund fragmentation model. It is shown how simple quantization rules combined with the causality considerations in the string fragmentation process describe the mass hierarchy of light pseudoscalar mesons. The quantized helix string model predicts observable quantum effects related to the threshold behaviour of the intrinsic transverse momentum of hadrons, and of the minimal transverse momentum difference between adjacent hadrons. The numerical fit of the properties of the QCD field breaking into ground state hadrons agree with astonishing precision with values obtained recently in the theory of knotted chromoelectric flux tubes.
\end{abstract}

PACS numbers: 11.25.-w, 03.75.Lm, 12.40.Yx, 12.38.-t

\section{INTRODUCTION}

The concept of the QCD string with a helical structure has been introduced in [1] and some of its potential explored in [2]. The model has been shown to decribe the experimentally established correlations between the longitudinal and transverse momentum components of hadrons measured by DELPHI at LEP [3] and the azimuthal ordering of hadrons, recently observed by ATLAS at LHC ([4]).

The aim of the present paper is to discuss in some detail the space-time evolution of partons following a breakup of a QCD string with a helix structure. A concept of string quantization emerges from these considerations which has the merit to describe, in a consistent manner, several experimental observations.

The paper is organized as follows: section II describes the transformation of light-cone coordinates used by the Lund string model for the case of 3-dimensional string topology and introduces the notion of causality in the string fragmentation process. Section III deals with the string quantization and the discrete mass spectrum of light mesons. Section IV investigates the threshold effect in the transverse momentum of direct hadrons. Section V uses the emerging quantization model to study the contribution of the intermediate resonant hadronic states to the measured particle correlation spectra. In section VI, the similarities between the helix string model and the theory of knotted chromoelectric flux tubes are discussed. Section VII closes the paper with a short summary.

\section{SPACE-TIME PROPERTIES OF HELICAL STRING MODEL}

In the transition from the 1-dimensional Lund string to a 3-dimensional helix-shaped string, it is necessary to reconsider some of the model properties. The basic assumption of a string modelling the confining QCD field with a constant string tension $(\kappa \sim 1 \mathrm{GeV} / \mathrm{fm})$ remains unchanged. However, the use of light-cone coordinates is no longer appropriate, as

\footnotetext{
* sarka.todorova@cern.ch
}

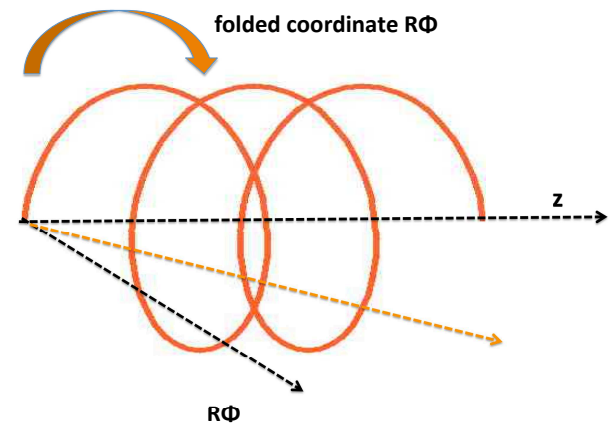

FIG. 1. The 2-dimensional coordinate system describing the motion along a helix-shaped string consists of the longitudinal string axis $z$ and the folded transverse coordinate $R \Phi$, where $R$ stands for radius of the helix and the $\Phi$ indicates the helix phase.

the trajectory of partons in the model is allways bended by the interaction with the field.

In the case of slowly varying field, the string can be approximated by an ideal helix with radius $\mathrm{R}$ and constant pitch $\mathrm{d} \Phi / \mathrm{dz}$, where $\Phi$ stands for the azimuthal angle (helix phase) and $z$ is the space coordinate parallel to the string axis. Movement of a parton along the string can be thus described with the help of the longitudinal coordinate $z$ and the folded transverse coordinate $\mathrm{R} \Phi$ (Fig. 11).

Following a string breakup at $\left[R e^{i \Phi_{B}}, z_{B}, t_{B}\right]$ into a pair of massless partons created at rest, the partons will move along the string and acquire the momentum

$$
\begin{aligned}
& p_{\|}(t)= \pm \kappa \beta c\left(t-t_{B}\right) \\
& p_{T}(t)= \pm \kappa R\left(e^{i \omega c\left(t-t_{B}\right)}-e^{i \Phi_{B}}\right)
\end{aligned}
$$

The longitudinal velocity of partons $\beta$ is related to the angular velocity $\omega$

$$
\beta=\sqrt{1-(R \omega)^{2}}
$$

( the light-cone coordinates are recovered in the limit case $\mathrm{R} \omega=0$ ). 
The momentum of a direct hadron created by adjacent string breakups at $\left[R e^{i \Phi_{i}}, z_{i}, t_{i}\right],\left[R e^{i \Phi_{j}}, z_{j}, t_{j}\right]$ is

$$
\begin{aligned}
E_{h} & =\frac{\kappa}{\beta}\left|\left(z_{i}-z_{j}\right)\right|=\frac{\kappa}{\beta}|\Delta z|, \\
p_{h, \|} & =\kappa \beta\left(t_{i}-t_{j}\right)=\kappa \beta \Delta t, \\
p_{h, T} & =\kappa R\left(e^{i \Phi_{i}}-e^{i \Phi_{j}}\right),
\end{aligned}
$$

and its mass is

$$
m_{h}=\kappa \sqrt{(\Delta z / \beta)^{2}-(\beta \Delta t)^{2}-(2 R \sin \Delta \Phi / 2)^{2}} .
$$

There is a fundamental difference (well illustrated by Eq. 4) between the helical string model and the standard Lund string model in what concerns the causality relation between breakup vertices.

In the standard Lund string model, the creation of a massive direct hadron requires a space-like distance between breakup vertices $\left(\beta=1, m_{h}>0 \Rightarrow|\Delta z|>|\Delta t|\right)$. The mass spectrum of hadrons is included in the model with the help of external parameters. Arguably, it is the absence of the cross-talk between hadron-generating adjacent string breakups which prevents the model from developing physics scenarios investigating the origin of the hadron mass hierarchy.

The situation is different in the case of the helical string model, where a time-like distance between breakup vertices is possible. As shown below, the causality considerations seem to pave the way to a better understanding of the role of quantum effects in the fragmentation. In order to explore the causal properties of the model, the time-like distance between adjacent breakup vertices will be imposed and the consequences studied. ( This does not necessarily mean the space-like separation between breakup vertices should not occur in the fragmentation - see the discussion in Section VI. )

There is an ambiguity concerning the way the signal is allowed to propagate. If the information ( about a breakup of the string at a given point) is allowed to pass along the string only, the space-time distance between adjacent vertices becomes negligible (to the extent we have neglected the parton masses) which means the propagating parton essentially triggers the following break-up and the mass of the outcoming hadron is (note that in this case $\Delta z=\beta \Delta t$ )

$$
m_{S}(\Delta \Phi)=\kappa R \sqrt{(\Delta \Phi)^{2}-(2 \sin \Delta \Phi / 2)^{2}} .
$$

It is interesting to see that the longitudinal momentum is factorized out from the equation and that the hadron mass depends on the transverse properties of the string shape only. To obtain a discrete mass spectrum, it is sufficient to introduce quantization of the transverse coordinate $\mathrm{R} \Phi$ (to be discussed in the following section).

There is of course also a possibility that the information about the breakup travels inside the string vortex (Fig. 2). To maintain the time-like difference between string breakups in such a case, the allowed time difference is then restricted to the interval

$$
\sqrt{(\Delta z)^{2}+(2 R \sin \Delta \Phi / 2)^{2}} \leq c \Delta t \leq \sqrt{(\Delta z)^{2}+(R \Delta \Phi)^{2}}
$$

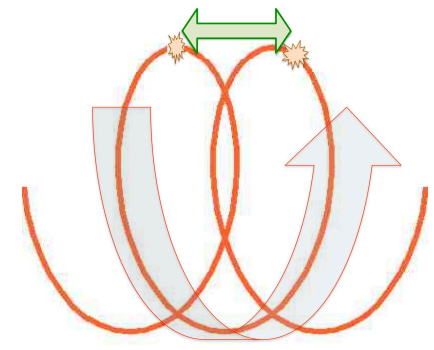

FIG. 2. The information about the string breakup propagates preferably along the string field, though a cross-talk between string loops is not excluded, either. The requirement of causal relation between breakups leads to an effective decoupling of the longitudinal and transverse component of the hadron momenta in the former case (see text).

and the outcoming hadron has a mass $m_{C}$ in the range

$$
m_{S}(\Delta \Phi) \leq m_{C}(\Delta \Phi) \leq m_{S}(\Delta \Phi) \sqrt{1+\beta^{2}}
$$

(the subscripts S,C stand for "singular" and "continuous" mass solutions).

The transverse energy $E_{T}$ of the hadron, which coincides with the transverse mass $m_{T}=\sqrt{m^{2}+p_{T}^{2}}$ in the "singular" case, does not depend on the choice (or the abandon) of the causality scheme and follows the simple relation

$$
E_{T}(\Delta \Phi)=\kappa R \Delta \Phi
$$

\section{MASS SPECTRA}

Building on the causality requirements, we have obtained relations between the transverse string properties and the allowed hadron mass spectrum. It seems only natural to take a step further and to try to establish a quantization pattern for the string fragmentation which would match the measured discrete hadron mass spectra.

Let's assume the string quantization is realized through the quantization of the transverse coordinate

$$
R \Phi \Rightarrow \mathrm{nR} \Delta \Phi=\mathrm{n} \xi,(\mathrm{n}=1,2, . .)
$$

and that the $\mathrm{n}=1$ case corresponds to the lightest hadron, the $\pi$ meson.

Eq. 5 is particularly interesting for the study of light meson mass hierarchy because it describes the narrow pseudoscalar states (PS) decaying into an odd number of pions

$$
\begin{aligned}
P S & \rightarrow \mathrm{n} \pi, \mathrm{n}=(1), 3,5, . . \\
m(P S) & =\kappa \sqrt{(\mathrm{n} \xi)^{2}-(2 \xi / \Delta \Phi)^{2} \sin ^{2}(\mathrm{n} \Delta \Phi / 2) .}
\end{aligned}
$$

The results of the best fit matching the Eq. 10 to experimentally measured data [5] are listed in Table 1. Despite 


\begin{tabular}{|c|c|c|}
\hline$\kappa \xi[\mathrm{MeV}]$ & $\kappa \mathrm{R}[\mathrm{MeV}]$ & $\Delta \Phi$ \\
\hline $192.5 \pm 0.5$ & $68 \pm 2$ & $2.82 \pm 0.06$ \\
\hline \hline meson & PDG mass [MeV] & model estimate $[\mathrm{MeV}]$ \\
\hline$\pi$ & $135-140$ & 137 \\
$\eta$ & 548 & 565 \\
$\eta^{\prime}$ & 958 & 958 \\
\hline
\end{tabular}

TABLE I. Best fit of the parameters of the pion ground state obtained from the mass spectrum of light pseudoscalar mesons. The $\eta$ mass is reproduced within a $3 \%$ margin which serves as the base of uncertainty for $R, \Delta \Phi$ parameters.

the fact that the simultaneous fit of 2 unknowns $(R, \Delta \Phi)$ from 3 hadronic states is overconstrained, a common solution describing the properties of the ground state is found. The $\pi$, $\eta(548)$ and $\eta^{\prime}$ (958) masses are reproduced by Eq. 10 with precision better than $3 \%$ using $\xi=0.192 \mathrm{fm}$ and $\Delta \Phi=2.8$ (for $\kappa=1 \mathrm{GeV} / \mathrm{fm}$ ).

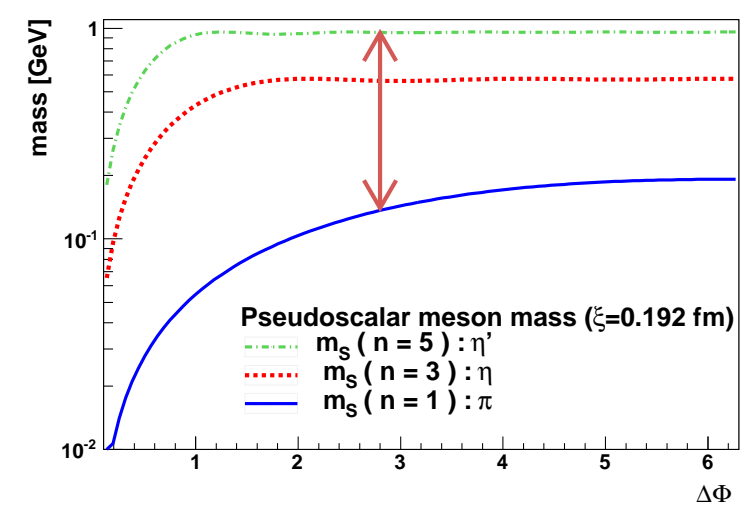

FIG. 3. The predicted masses of light pseudoscalar mesons as function of helix phase difference $\Delta \Phi$, for fixed $R \Delta \Phi=0.192 \mathrm{fm}$ rad.

Fig. 3 shows the dependence of the mass of PS mesons as a function of $\Delta \Phi$ in Eq.(10). With increasing $\Delta \Phi$, the predicted masses of $\eta$ and $\eta^{\prime}$ reach the plateau (around $\Delta \Phi \sim 1.5 \mathrm{rad}$ ) and lose sensitivity to the $\Delta \Phi$ value, but the mass of the $\pi$ meson rises steadily till $\Delta \Phi \sim 5 \mathrm{rad}$ and effectively fixes the $\Delta \Phi$ value in the model.

The scalar nature of PS states is in agreement with the expectations of the quantization model: $m_{T}(P S)=\mathrm{n} m_{T}(\pi)$, thus the decay products of $\left(\eta, \eta^{\prime}\right)$ have negligible (longitudinal) relative momentum in the rest frame of the mother resonance.

If the quantization model, in the first approximation, fits the mass spectra of light PS mesons, what can be said about the vector mesons (VM)?

The lightest vector mesons $\rho(770)$ and $\omega(782)$ can be interpreted as $\mathrm{n}=4$ states decaying into $\mathrm{m}<\mathrm{n}$ pions:

$$
m_{S}(n=4)=0.76 \mathrm{GeV}
$$

or $\mathrm{n}=3$ states formed according to Eq. 7

$$
\sqrt{2} m_{S}(n=3) \sim 0.79 \mathrm{GeV},
$$

and their non-zero total angular momentum arises from the relative momentum of decay products (kinematically allowed since $m_{T}(V M)>\mathrm{m} m_{T}(\pi)$.

The mass of $K^{*}(890)$ and $\Phi(1020)$ mesons can be roughly associated with the mass of the $\rho(770)$ increased by the mass of the strange quark(s) $(\sim 120 \mathrm{MeV})$. (The same reasoning would classify $\mathrm{K}$ meson as a $n=2$ state).

The tentative classification of VM hadron states mentioned above is more an illustration than a prediction ( a more precise relation between the total angular momentum of the hadron and the field properties has yet to be established ). It is worth noticing however that the quantization of the transverse component of the string is equivalent to the quantization of the angular momentum $J$ stored in the string ( proportional to the transverse area spanned by the string ) and that the relation

$$
J \simeq \kappa(R \Delta \Phi)^{2}=m_{T}^{2} / \kappa
$$

indicates that the spectra derived from the model will lie along Regge trajectories [6].

\section{TRANSVERSE MOMENTUM THRESHOLD}

The discretization of the mass spectrum is not the only quantum effect which can be observed in the string fragmentation. In fact, the current investigation of the properties of the helix string quantization was prompted by the study of the inclusive $p_{T}$ spectra. In [2] it has been shown that the helix string model significantly improves the description of the inclusive low $p_{T}$ region. It has been also shown that the strength of the azimuthal correlations between hadrons can be described by the model only if the helix string model is extended to the decay of short-lived resonances. However, it turns out that such an extension spoils the agreement between the LEP data and the helix model essentially because the resonance decay according to the helix shaped "field memory" produces way too many low $p_{T}$ particles (Fig. (4).

The effect cannot be tuned away as there are essentially no relevant free parameters left in the model. A careful study of the discrepancy and the gradients of the $p_{T}$ spectra does not exclude existence of a natural $p_{T}$ cutoff just below $0.2 \mathrm{GeV}$ (Fig. 4, bottom plot).

It is therefore encouraging to see - on the basis of results obtained in the previous section - that the production of soft pions with the $p \sim p_{T}<0.14 \mathrm{GeV}$ should be supressed in the quantized model. This result has yet to be propagated through the entire fragmentation and decay chain but this particular model feature is expected to help the regularization of the soft particle production in the helix string model extended to the decay of resonances. 

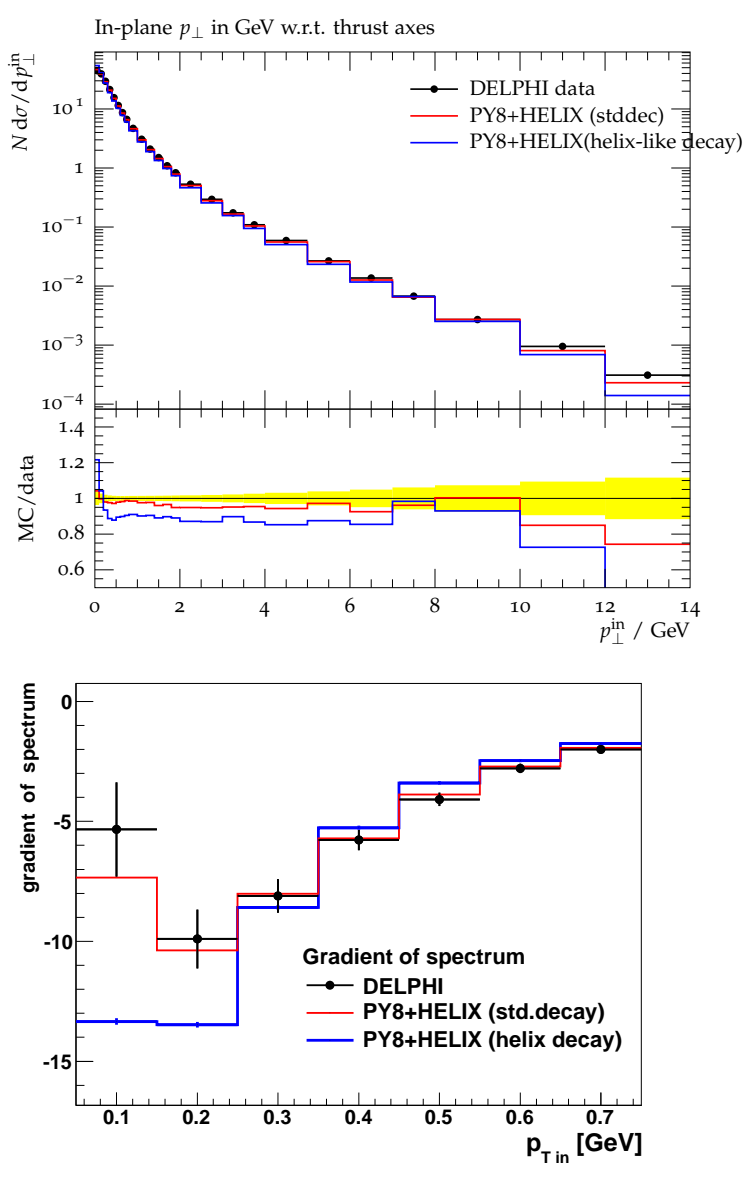

FIG. 4. Comparison of the helix string model predictions with the DELPHI data [3]. Upper plot: The production of low $p_{T}$ charged particles is overestimated by the model when decay of short lived resonances is treated as a smooth continuation of the fragmentation of the helix string. Lower plot : Comparison of gradients of the inclusive distributions. The extended helix string model overestimates the rate of particles with low $p_{T}$. The most significant change of slope occurs in the data below $p_{T} \sim 0.2 \mathrm{GeV}$ - this may be related to the existence of an intrinsic pT cutoff.

\section{MOMENTUM DIFFERENCE OF ADJACENT HADRONS}

The quantization of the helix string implies a quantization of the momentum difference between adjacent hadrons. Since the local charge conservation forbids the production of adjacent like-sign charged hadron pairs in the fragmentation process, the quantum effects can play a large role in the correlation phenomena with a significant difference between particle pairs with like-sign and unlike-sign charge combination.

In the approximation of an ideal, or slowly varying helix string field, it is possible to make an estimate of the charge combination asymmetry induced by quantization. Consider a chain of adjacent charged pions in the ground state, for example from $\eta^{\prime}$ decay. The homogenity of the QCD field implies the difference between longitudinal momenta components of such pions is negligible. The momentum difference between

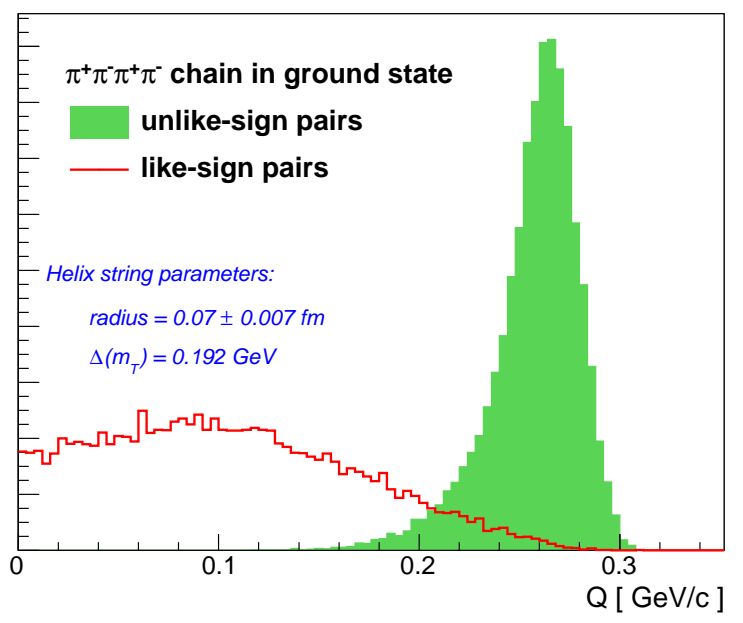

FIG. 5. The correlation pattern estimate for a chain of 4 charged pions in the ground state (Table 1). The longitudinal momentum differences are neglected, a variation of $10 \%$ is applied on the helix radius instead, in order to obtain a smooth spectra.

pions along the chain is then given by the helix phase difference

$$
\begin{aligned}
Q & =\sqrt{-\left(p_{i}-p_{j}\right)^{2}} \approx 2 p_{T}\left|\sin \left[0.5\left(\Phi_{\mathrm{i}}-\Phi_{\mathrm{j}}\right)\right]\right| \\
& =2 p_{T}|\sin [0.5(\mathrm{j}-\mathrm{i}) \Delta \Phi]|
\end{aligned}
$$

where $p_{T}(\sim 0.14 \mathrm{GeV})$ is the transverse momentum of the pion in the ground state, $i, j=0,1,2,3$ are integers corresponding to the rank of the hadron along the chain, and $\Delta \Phi(\sim 2.8)$ is the opening azimuthal angle between adjacent ground state pions. Fig. 5 shows the resulting correlation pattern with a marked separation of like-sign and unlike-sign pairs (the helix radius has been randomly varied by $10 \%$ in order to produce a smooth spectrum). The onset of excess of like-sign pairs occurs at $Q \sim 0.2 \mathrm{GeV}$ in the model. A large amount of experimental data provides evidence of an excess of like-sign hadron pair production in the low Q region. Most often, the data are studied from the perspective of the Handbury-Brown-Twiss model, i.e. as a signature of the incoherent particle production. The helix string model suggests an alternative point of view - such correlations may well be associated with fully coherent hadron production. In the specific case under study, due to the large opening angle $\Delta \Phi$, the quantized chain of ground state pions acquires properties reminiscent of Bose-Einstein condensate.

It should be possible to make a more precise experimental evaluation of the role of hadron 'chains' (and $\eta^{\prime}$ decay) in the correlation signal. Such a study may have a significant impact on the further development of the helix model, as it may confirm, or reject, the hypothesis of a strong link between resonance production and correlation phenomena. 


\section{DISCUSSION OF RESULTS}

The radius of the quantized helix string obtained in Section III is significantly smaller than the tuned radius of the nonquantized helix string and will require further adjustments in the fragmentation model in order to describe the data ( via radial string excitations, gradual field relaxation, or adjustment of the parton shower cutoff ). On the other hand, it is fair to admit that, though somewhat surprising, the numerical values describing the ground hadronic state do provide a basis for explanation of the charged combination asymmetry in 2-particle correlations, and to the regularization of the soft particle production, without introduction of additional free parameters in the model.

Moreover, the estimated effective radial size of the helix string breaking into a set of ground state hadrons $(68 \pm 2 \mathrm{MeV}$ ) turns out to be in agreement with the estimate of the confinment scale $\Lambda_{\text {tube }}=65.16 \pm 0.61 \mathrm{MeV}$ obtained from the fit of $\mathrm{J}^{++}$spectra modelled via tight topological QCD knots [7].

The agreement is remarkable not only because the two studies use a different type of input data ( mesons/open strings versus glueballs/closed strings ) but also due to the fact that reasoning behind the two semi-classical models follows distinct paths, one [1] being based on study of the gluon emission, the other [7] stemming from the physics of continuum (plasma) and topological properties of QCD flux tubes. In both models, the helicity conservation plays a central role, and the approach to the problematics seems to be fairly consistent up to the point that a certain aspects of one model can be intuitively understood in terms of the alternative approach. For example, the secondary emission of gluons in the helix string model, which leads to the homogenization of the string field (prefered by the data), can possibly be seen as the "field relax- ation into equilibrium state with minimal energy" - the term employed by the model of knotted QCD flux tubes.

As a final remarque, it should be emphasized the helix string model is expected to remain conformant to the string area law governing the string breakup probability. That means the model should be able to handle the fragmentation resulting from several independent ( uncorrelated, space-like ) string breakups followed by a chain of correlated breakups.

\section{CONCLUSIONS}

The properties of the quantized helix string model have been investigated using a data driven simple quantization recipe. The model allows to introduce proper causal relations between the breakup vertices in the string fragmentation. The causality represents a strong constraint for the particle production and helps to understand the emergence of narrow hadronic resonant states. The fit of the light pseudoscalar mesons provides the parameters describing the ground hadronic state, and allows to make predictions concerning the threshold behaviour of relevant observables, to be verified with the help of experimental data. Last but not least, the fitted estimate of the helix string radius $(\kappa \mathrm{R}=68+-2 \mathrm{MeV})$ is found to be in good agreement with the results of a recent study [7] of mass spectrum of $J^{++}$states (glueball candidates) in the frame of the model of knotted QCD flux tubes.

\section{ACKNOWLEDMENTS}

The author would like to thank Prof.J.Bjørken for valuable suggestions.
[1] B. Andersson et al., J. High Energy Phys. 09, 014 (1998).

[2] Š.Todorova, Phys.Rev.D 86, 034001 (2012).

[3] DELPHI Collaboration, Z.Phys. C73, 11 (1996), Rivet analysis:DELPHI-1996-S3430090.
[4] ATLAS Coll., Phys.Rev.D 86, $052005 \quad$ (2012), Rivet analysis:ATLAS-2012-I1091481.

[5] Particle Data Group, Phys.Rev.D 86, 010001 (2012).

[6] R. P. Irving, A. C.; Worden, Phys.Rep. 34 (3), 117-231 (1977).

[7] R.V. Buniy et al., (2012), arXiv : 1212.1500[hep - ph]. 